\title{
Enhanced antagonism of BST-2 by a neurovirulent SIV envelope
}

\author{
Kenta Matsuda, ${ }^{1}$ Chia-Yen Chen, ${ }^{1}$ Sonya Whitted, ${ }^{1}$ Elena Chertova, ${ }^{2}$ David J. Roser, ${ }^{2}$ Fan Wu, ${ }^{1}$ Ronald J. Plishka, ${ }^{1}$ Inour Ourmanov, \\ Alicia Buckler-White, ${ }^{1}$ Jeffrey D. Lifson, ${ }^{2}$ Klaus Strebel, ${ }^{1}$ and Vanessa M. Hirsch ${ }^{1}$ \\ 'Laboratory of Molecular Microbiology, National Institute of Allergy and Infectious Diseases (NIAID), NIH, Bethesda, Maryland, USA. ${ }^{2}$ AIDS and Cancer Virus Program, Leidos Biomedical Research Inc., \\ Frederick National Laboratory for Cancer Research, Frederick, Maryland, USA.
}

\begin{abstract}
Current antiretroviral therapy (ART) is not sufficient to completely suppress disease progression in the CNS, as indicated by the rising incidence of HIV-1-associated neurocognitive disorders (HAND) among infected individuals on ART. It is not clear why some HIV-1-infected patients develop HAND, despite effective repression of viral replication in the circulation. SIV-infected nonhuman primate models are widely used to dissect the mechanisms of viral pathogenesis in the CNS. Here, we identified 4 amino acid substitutions in the cytoplasmic tail of viral envelope glycoprotein gp41 of the neurovirulent virus SIVsm804E that enhance replication in macrophages and associate with enhanced antagonism of the host restriction factor BM stromal cell antigen 2 (BST-2). Rhesus macaques were inoculated with a variant of the parental virus SIVsmE543-3 that had been engineered to contain the 4 amino acid substitutions present in gp41 of SIVsm804E. Compared with WT virusinfected controls, animals infected with mutant virus exhibited higher viral load in cerebrospinal fluid. Together, these results are consistent with a potential role for BST-2 in the CNS microenvironment and suggest that BST- 2 antagonists may serve as a possible target for countermeasures against HAND.
\end{abstract}

\section{Introduction}

Prior to development of antiretroviral drugs, infection by HIV-1 frequently led to development of HIV-associated dementia (HAD) in infected individuals (1). HAD can be accompanied by neuropathological abnormalities referred to as HIV-1 encephalitis (HIVE), which in severe cases can lead to a vegetative state and death. Although antiretroviral monotherapy (ART) had limited impact, possibly due to emergence of resistant virus $(2,3)$, development and administration of combinatorial antiretroviral therapy (cART) has led to a dramatic reduction in the incidence of such neurological disorders, at least among individuals who can afford and adhere to the treatment schedule. However, recent studies revealed an increased incidence in HIV-1-associated neurocognitive disorder (HAND) or minor cognitive and motor disorder (MCMD) among cART-treated individuals, suggesting that some level of disease progression continues regardless of effective suppression of viral replication in the circulation by cART, and it is a major concern (4).

The mechanisms of disease progression in the CNS under successful suppression of peripheral viral replication are still unclear. One potential explanation is the incomplete suppression of viral replication by ART, suggested by the emergence of "blips" of plasma viremia during treatment, causing direct neurological damage by viral replication $(5,6)$. It is also widely accepted that some antiretroviral drugs show poor efficacy in the CNS due to their inability to penetrate the blood brain barrier (BBB) (7), which could make CNS a sanctuary of viral repli-

Authorship note: K. Matsuda and C.Y. Chen contributed equally to this work. Conflict of interest: The authors have declared that no conflict of interest exists. Submitted: July 13, 2015; Accepted: March 22, 2016.

Reference information: J Clin Invest. 2016;126(6):2295-2307. doi:10.1172/JCI83725. cation. Indeed, multiple studies show persistent viral replication in the CNS of infected individuals, despite successful suppression of viral replication in the circulation $(8,9)$. Other reports suggest that CNS pathology could be the result of chronic immune activation during cART, causing bystander cell death in the CNS $(10,11)$. In addition, some reports suggest direct toxicity of antiretroviral drugs in the CNS $(12,13)$.

To clarify the mechanisms leading to HAD and HAND/ MCMD and to develop new strategies for augmenting the efficacy of cART for prevention of CNS sequelae, our group has been developing a nonhuman primate model of neuroAIDS. Although it may not directly reflect the disease progression in HIV-1-infected individuals, nonhuman primate models enable access to CNS samples at all stages of disease progression, something that cannot be done with human patients for ethical reasons. Our group has recently reported on the isolation of a neurovirulent strain of simian immunodeficiency virus (SIV) African primate lentivirus (sm), SIVsm804E, that induced neuropathology in $83 \%$ of infected rhesus macaques when animals with restrictive MHC or TRIM alleles were eliminated from the cohort $(14,15)$. This virus isolate was generated by sequential passage of the nonneurovirulent parental virus SIVsmE543-3 through rhesus macaques. We therefore compared SIVsmE543-3 and SIVsm804E to try to delineate viral determinants of neurovirulence. Mutations that accumulated in viral accessory protein negative factor (nef) and the long terminal repeats (LTR) were first assessed, since there are reports suggesting that mutations in these regions can confer neurotropism and improve replication in the CNS (16-18). Additional reports suggest that Nef can exert direct toxicity in certain cell populations in the CNS, such as astrocytes $(19,20)$. Hence, a chimeric SIVsmE543-3 clone with a portion of the $3^{\prime}$ gp 41 cytoplasmic tail, nef, and $3^{\prime}$-LTR 
from SIVsm804E was created to investigate the impact of nef and LTR mutations in vitro.

It has been suggested that macrophages/microglia are the primary targets of HIV-1/SIV infection and replication in the CNS; therefore, macrophage tropism and replication of virus in myeloid cells would appear to be critical for development of neurological disorders (21). This is further supported by the fact that most HIV-1 isolates from the CNS from subjects with neurocognitive disorders are macrophage tropic (22). Indeed, the main difference between SIVsmE543-3 and SIVsm804E is that the latter virus replicates more efficiently in monocyte-derived macrophages (MDMs) (15). Since macrophage tropism is strongly influenced by the number of glycosylation sites on the envelope (Env) glycoprotein, mostly within gp120 (23), it was hypothesized that mutations found in nef and LTR of SIVsm804E would have minimal impact on SIVsmE543-3 replication in MDMs. However, the chimeric clone unexpectedly showed enhanced replication in macrophages. In the present study, we determined the Env region of SIVsm804E to be responsible for conferring enhanced viral replication in macrophages. Here, we report that 4 amino acid substitutions at the gp41 cytoplasmic tail are associated with enhanced replication in MDM and are also associated with enhanced viral antagonism against the host restriction factor BM stromal cell antigen 2 (BST-2), which suggests BST-2 may play important roles in restricting viral replication in the CNS.

\section{Results}

SIVsmE543-3 with 3' gp41/nef/LTR from the neurovirulent SIVsm804E shows enhanced replication in MDM. To investigate the impact of mutations in nef and LTR on replication of SIVsmE543, SIVsm804E CL4E4, CL6E6, and CL7E7 were generated by introduction of the $3^{\prime} \mathrm{gp} 41 / n e f / L T R$ region of SIVsm804E into the corresponding region of SIVsmE543-3 (Figure 1A). SIVmac239, a virus that replicates primarily in PBMC but not MDM, was used as a control. PBMCs and MDMs were infected with these chimeric viruses, and reverse transcriptase (RT) activity of the culture supernatant was monitored to measure the level of viral replication. The original SIVsm804E and SIVsmE543-3 showed comparable replication in PBMC, although SIVsm804E showed slightly higher RT activity at 12 days after infection. The chimeric clones showed comparable levels of replication with SIVsmE543-3. SIVmac239 showed robust replication in PBMCs with delayed peak viral replication compared with SIVsmE543 variants. In contrast, while SIVsm804E showed robust replication in MDM, SIVsmE543-3 showed low levels of replication, consistent with our previous report (15). As expected, SIVmac239 showed no measurable replication in MDM. Surprisingly, all of the chimeric viruses showed significantly higher replication in MDM compared with SIVsmE543-3 (4E4, $P=0.0001$; 6E6, $P=0.0029$; and 7E7, $P=0.0026$ ), indicating that the $3^{\prime} \mathrm{gp} 41 /$ nef/ LTR region introduced from SIVsm804E conferred enhanced replication in macrophages (Figure 1, B and C).

Identification of amino acid substitutions responsible for enhanced replication in macrophages. To identify the region(s) responsible for the enhanced replication of SIVsm804E in macrophages, 2 additional chimeric clones were constructed by introducing nef and/ or 3' gp41 from SIVsm804E CL7E7 into the original SIVsmE543-3, and these were designated SIVsmE543 804CTN and SIVsmE543 $804 \mathrm{CT}$, respectively (Figure 2A). Replication profiles of the 3 chimeric clones were comparable with those of SIVsmE543-3 in PBMCs. However, all of the chimeric clones containing 3' gp 41 from SIVsm804E showed enhanced replication in MDMs (804CTN, $P=0.0044$; 804ECT, $P=0.0032$ ), indicating that mutation(s) in the $3^{\prime}$ region of gp41 were responsible for the enhanced replication in macrophages (Figure 2, B and C). To further determine which specific mutation(s) were responsible for the enhanced replication, the 3' gp41 portion of SIVsm804E CL4E4, CL6E6, and CL7E7 were sequenced, and amino acid sequences were aligned. Since enhanced replication in macrophages was observed consistently among all 3 chimeric clones, amino acid substitutions that were conserved among the 3 clones were thought to be responsible. As a result, 4 amino acid substitutions I805T, I828R, T829A, and V878I were identified (Figure 3A). To test which of those 4 amino acid substitutions was responsible for the enhanced replication, we introduced single amino acid substitutions into SIVsmE543-3 by site-directed mutagenesis (Figure 3B), and replication of those 4 mutants was assessed in PBMC and MDM. Consistent with the results from Figure 2B, replication of SIVsmE543-3 and all 4 mutants in PBMC were similar. However, surprisingly, all mutants also showed enhanced replication in MDM. Although the increase observed for 543CT\#1 and 543CT\#2 was modest, other comparisons showed statistically significant differences ( $\# 3, P=0.0025 ; \# 4, P=0.0352 ; \# 154$, $P=0.0141)$. This result indicates that all 4 mutations conferred improved replication in macrophages to various degrees, apparently independent from one another (Figure 4, A and B).

Assessment of virion-incorporated Env levels. Reports suggest that truncation of gp41 cytoplasmic tail increases virion incorporation of Env, which may enhance viral replication in macrophages by increased fusion activity (24-26). Although the mutations in the present study (I805T, I828R, T829A, and V878I) did not result in truncation, they affected the cytoplasmic tail of gp 41. Therefore, to assess if these 4 amino acid substitutions affected the incorporation of Env glycoproteins by virions, SIVsmE543-3 and SIVsmE543CT clones were purified, concentrated, and examined by SDS-PAGE using a dual-color fluorescent imaging method (Figure 5A), and the ratio of virion p27 content against virion gp120 content was calculated (Figure 5B and refs. 27, 28). The levels of virion-incorporated Env protein between SIVsmE543-3 and SIVsmE543CT clones were not significantly different, indicating that the mutations we have identified as enhancing replication in macrophages do not influence the virion incorporation of Env. Immunoblot analysis was also consistent with this interpretation (Supplemental Figure 1; supplemental material available online with this article; doi:10.1172/JCI83725DS1).

Assessment of viral release efficiency/BST-2 antagonism. Apart from the potential influence of gp41 truncations on the level of virion-incorporated Env glycoproteins, mutations in the gp41 cytoplasmic tail were reported to affect viral antagonism of BST-2 (also known as tetherin or CD317) (29). BST-2 is a host factor that restricts the release of nascent viral particles from the surface of infected cells $(30,31)$. Previous reports have shown that most SIVs utilize their Nef proteins to antagonize BST-2 restriction (32), but some SIVs (e.g., SIV isolated from tantalus monkey [SIVtan]) utilize the Env protein (33). Therefore, pulse-chase assays were con- 
A

SIVsmE543
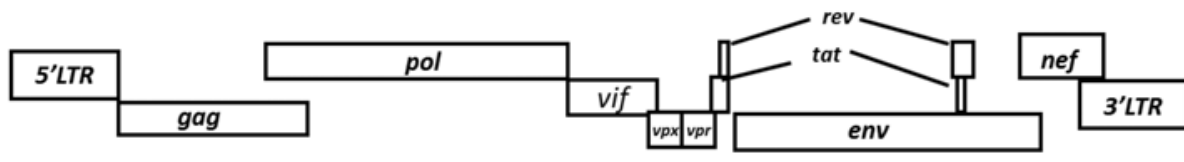

SIVsm804E
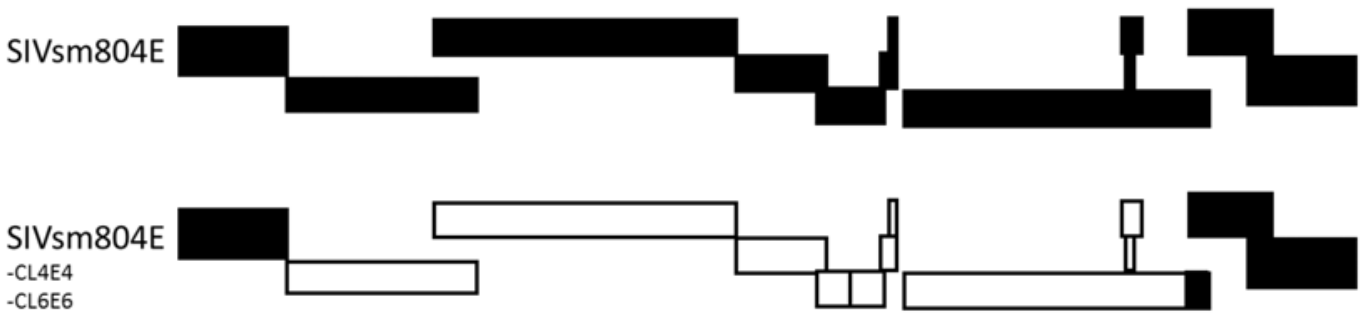

PBMC

B

MDM
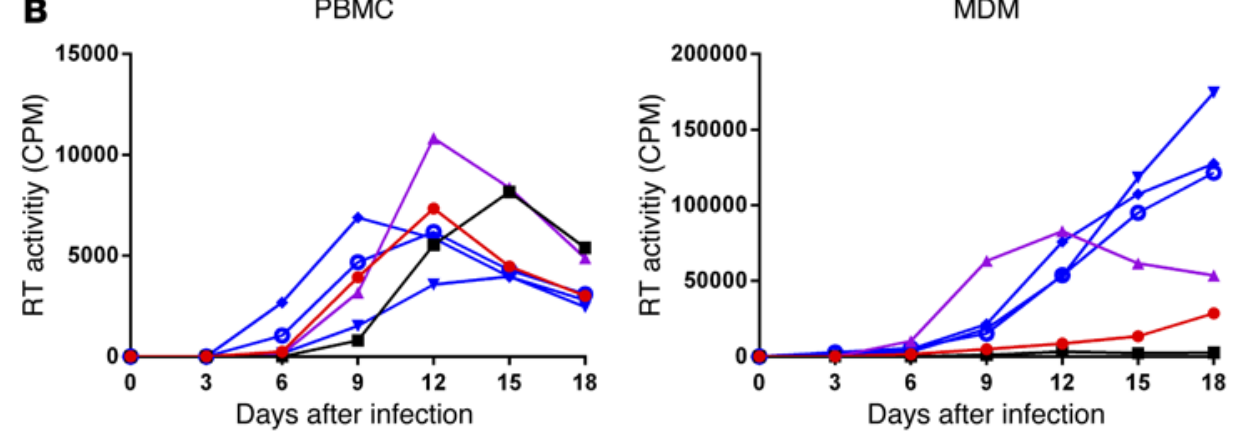

$\rightarrow$ SIVsmE543-3

- SIVmac239

- SIVsm804E

* CL4E4

$\rightarrow$ CL6E6

$\rightarrow$ CL7E7
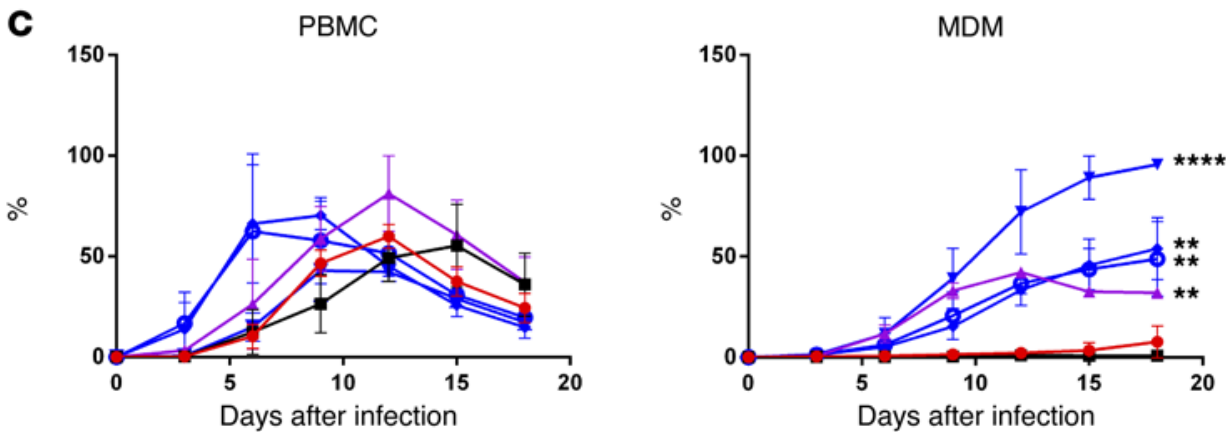

Figure 1. Viral replication in PBMC and MDM. (A) Schematic diagram of SIVsmE543-3, SIVsm804E, and 3 chimeric clones. White bars indicate open reading frames (ORF) from SIVsmE543-3; black bars indicate ORF from SIVsm804E. (B) Replication kinetics of SIVmac239, SIVsmE543-3, SIVsm804E, and 3 chimeric SIVsmE543-3 clones containing 3' gp41/nef/LTR from SIVsm804E on PBMCs and MDMs. Three independent experiments were conducted with PBMC and MDM obtained from donors RhDCCW and RhMO3. A representative experiment is shown (donor RhMO3). (C) Mean replication efficiency normalized as percentage of maximal replication activity reached for different cell preparations infected with indicated set of viruses are plotted. The bars represent the standard errors of the means of normalized values obtained on cells isolated from different macaques. ${ }^{* *} P \leq 0.01$ and ${ }^{* * * *} P \leq 0.0001,2$-way ANOVA (replication kinetics of chimeric clones compared with SIVsmE543).

ducted to first assess whether SIVsmE543-3 utilizes its Nef or Env protein to antagonize BST-2. The Env protein from HIV-2 isolate, ROD10 - which is known to antagonize human BST-2 (hBST-2) efficiently (34) - showed efficient antagonism against rhesus BST-2 (rBST-2). On the other hand, the Env protein from another HIV-2 isolate, ROD14 - which does not antagonize hBST-2 (35) - also did not antagonize rBST-2. These 2 Env proteins were used as positive and negative controls, respectively, for the characterization of our SIV proteins. As has been previously reported, SIVmac239 Nef showed efficient antagonism against rBST-2 $(P$ $=0.0001$ against ROD14) (36). However, it is noteworthy that, consistent with the previous report, the SIVmac239 Env protein also showed BST-2 antagonism $(P=0.0004$ against ROD14). In fact, SIVmac239 Nef and Env enhanced virus release to similar extents in our experimental setup (Figure 6). Thus, SIVmac239 can utilize both Nef and Env to antagonize BST-2. On the other hand, the SIVsmE543-3 Nef protein did not antagonize rBST-2 $(P=0.3607$ against ROD14) (Figure 6). Instead, SIVsmE543-3 appears to exclusively utilize its Env protein to antagonize BST-2 $(P=0.0001$ against ROD14).

Impact of mutations in the gp41 cytoplasmic tail on BST-2 antagonism. To assess if mutations in the gp41 cytoplasmic tail that enhance viral replication in macrophages have an impact on BST-2 antagonism, pulse-chase and immunoprecipitation 
A
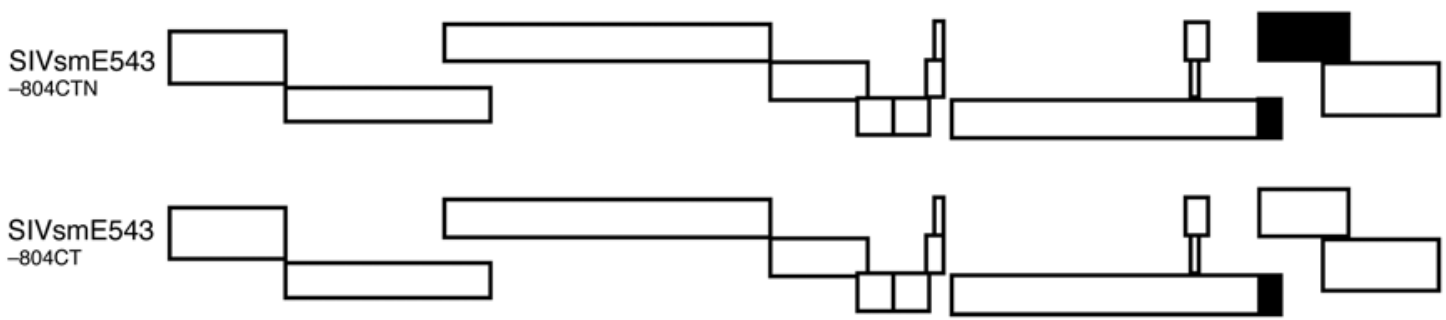

B

PBMC
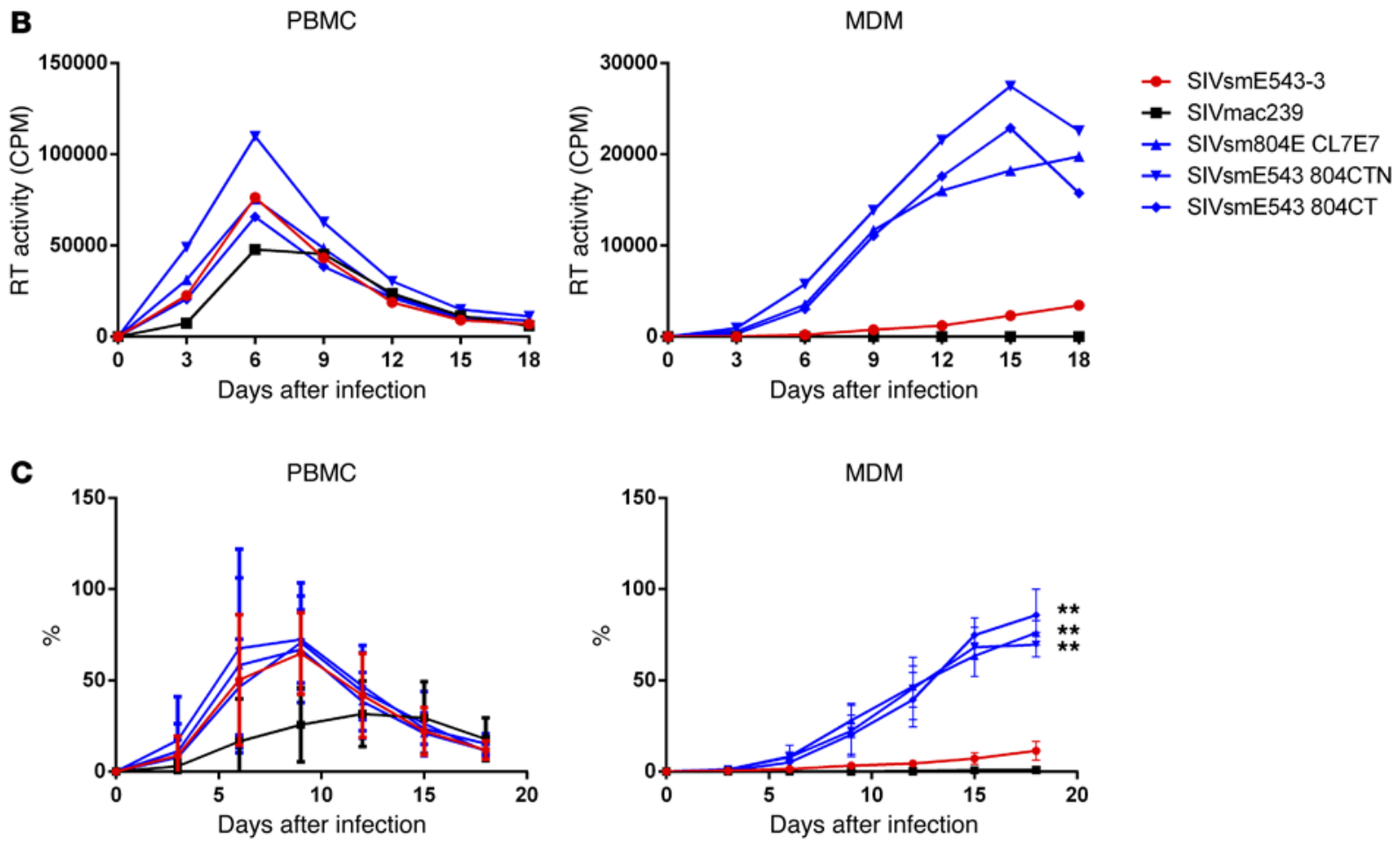

Figure 2. Determination of the region(s) responsible for enhanced replication in MDM. (A) A schematic diagram of the chimeric viruses is shown. White bars indicate ORFs from SIVsmE543-3; black bars indicate ORFs from SIVsm804E. (B) Replication kinetics of SIVmac239, SIVsmE543-3, SIVsm804E, and 3 chimeric SIVsmE543-3 clones containing various regions from SIVsm804E on PBMCs and MDMs. Three independent experiments were conducted with PBMC and MDM obtained from donors RhDCCW and RhMO3. A representative experiment is shown (donor RhMO3). (C) Means of replication efficiency normalized as percentage of maximal replication activity reached for different cell preparations infected with indicated set of viruses. The bars represent the standard errors of the means of normalized values obtained on cells isolated from different macaques. ${ }^{* *} P \leq 0.01,2$-way ANOVA (replication kinetics of chimeric clones compared with SIVsmE543).

assays were conducted on SIVsmE543-3 chimeras with single amino acid substitutions derived from SIVsm804E and with a clone carrying all 4 mutations. Consistent with the effect of each of these substitutions on replication in macrophages, each of the SIVsmE543-3 mutants with single amino acid substitutions (SIVsmE543CT\#1, SIVsmE543CT\#2, SIVsmE543CT\#3, and SIVsmE543CT\#4) showed statistically significant increases in virion release $(P=0.001, P=0.0049, P=0.0015$, and $P=$ 0.0004 against WT E543-3 Env, respectively) (Figure 7), indicating that each amino acid substitution can independently enhance BST-2 antagonism. A mutant with all amino acid substitutions (SIVsmE543CT\#154) showed comparable increase in viral release with the mutants with a single substitution $(P=$ 0.0004 against WT E543-3 Env), suggesting that the number of amino acid substitutions does not correlate with the degree of increase in viral release, at least in this in vitro assay (Figure 7).
This was consistent with the pattern of results for enhanced replication of these variants in MDM (Figure 4, A and B). The correlation of these cytoplasmic gp41 mutations with both enhanced BST-2 antagonisms and replication in MDM is highly suggestive of a causal relationship. However, we were unable to confirm causality using primary macrophages to measure virus release conferred by the gp41 substitutions due to technical difficulties in performing such assays in MDM.

To clarify whether the enhanced viral release of variants with gp41 cytoplasmic tail substitutions from 293T cells was genuinely the result of BST-2 antagonism, we conducted the same experiment in the absence of BST-2 (Supplemental Figure 2). In the absence of BST-2, the levels of virion release for the WT E543 Env and E543CT\#154 Env were comparable with each other, indicating that differences observed in the presence of rBST-2 in Figure $7 \mathrm{C}$ is genuinely the result of BST-2 antagonism by the Env variants. 


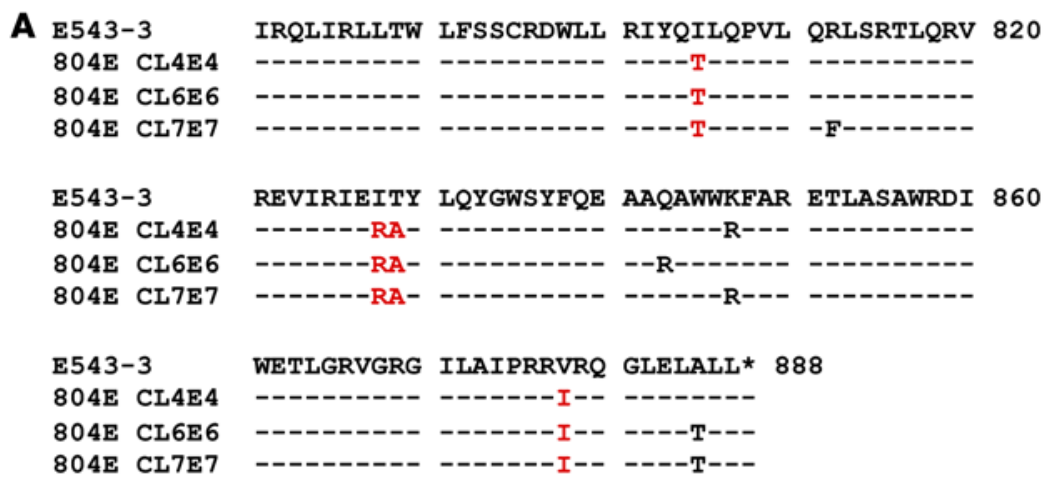

B

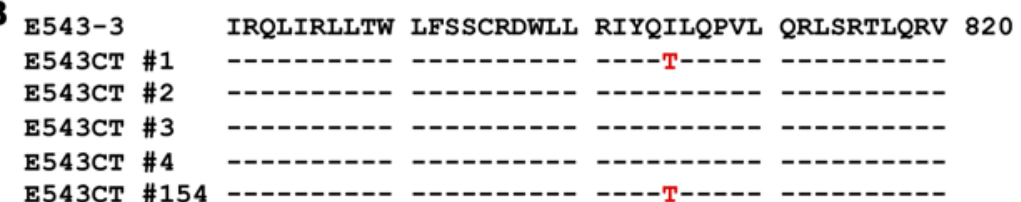

E543CT \#154

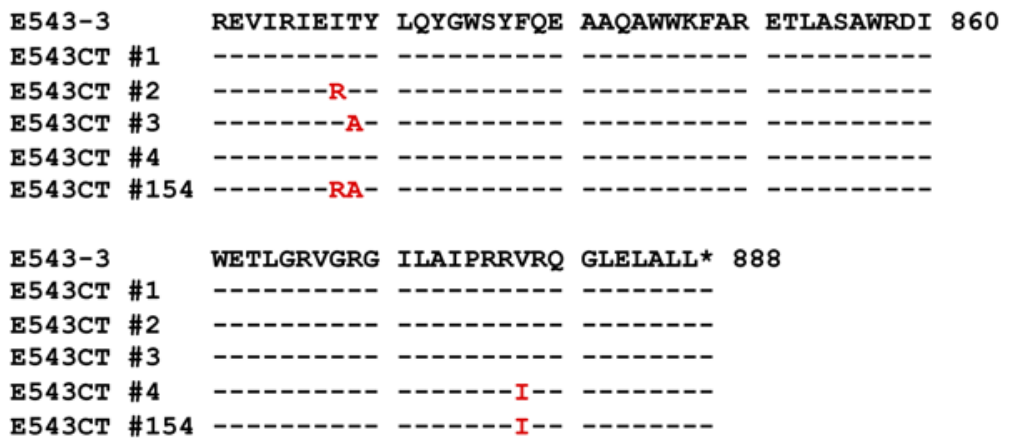

Figure 3. Determination of specific amino acid substitution(s) responsible for enhanced replication in MDM. (A) 3' gp41 amino acid sequences of 3 SIVsm804E clones. Amino acid substitutions conserved among all 3 clones are indicated by in red. (B) Amino acid sequences of 5 SIVsmE543-3 clones with point mutation(s) introduced in the $3^{\prime}$ gp 41 cytoplasmic tail. Amino acid substitution(s) are indicated in red. Asterisks indicate stop codons.

Gp41 sequences of historical viral stocks from serial passages. The neurovirulent virus SIVsm804E was isolated after 4 sequential passages of the nonneurovirulent parental virus SIVsmE543-3 through rhesus macaques. To determine the timing of acquisition of the 4 critical amino acid substitutions in $3^{\prime}$ gp 41 , amino acid sequence of viral stocks isolated during the sequential in vivo passage performed to derive SIVsm804E (passage 1, SIVsmH455; passage 2, SIVsmH631Br; passage 3, SIVsm783Br; and passage 4, SIVsm804E) were compared. Amino acid substitutions at positions I805T, I828R, T829A, and V878I accumulated through sequential passages. After the first passage, none of the SIVsmH445 variants contained mutations that we identified as responsible for the enhanced BST-2 antagonism. Those mutations appeared after the second passage, as 1 of 7 (14\%) SIVsmH631Br variants contained I805T and all variants contained the T829A mutation. All SIVsm783Br variants contained I805T and T829A mutations after the third passage, indicating that $1805 \mathrm{~T}$ was positively selected and stabilized within the population. Furthermore, 2 mutations - I828R and V878I - emerged in SIVsm783Br. All 12 variants contained I828R mutation, and 7 of 12 variants (58\%) contained V878I. Although a number of other mutations emerged in SIVsm804E after the final passage, frequencies of mutations I805T, I828R, T829A, and V878I are comparable with that of SIVsm783Br (Figure 8). Acquisition of these 4 mutations correlates well with the in vivo neuropathogenicity (15), suggesting that these mutations play an important role in disease progression in the CNS.

Replication of SIVsmE543CT\#154 in rhesus macaques. To assess the in vivo replication capacity and potential neurovirulence conferred by these 4 Env substitutions, 6 rhesus macaques (H888, H889, H890, H891, H882, and H893) were i.v. inoculated with SIVsmE543CT\#154, a clone that differs from the parental SIVsmE543 only in these 4 gp41 mutations. The kinetics of plasma and CSF viral load compared with 3 rhesus macaques (H625, H627, and H628) that had been i.v. inoculated with the parental virus SIVsmE543-3 as a nonneurovirulent control group. All of rhesus macaques used in this study shared the moderately susceptible TRIM5 $\alpha$ genotype (TFP/Q). As shown in Figure 9A, all infected animals showed comparable peak viremia in plasma at 2 weeks after infection $\left(3 \times 10^{5}\right.$ to $9 \times 10^{6}$ copies $\left./ \mathrm{ml}\right)$, with the exception of one animal, H890, with substantially lower peak viremia substantially $\left(6 \times 10^{4}\right.$ copies $\left./ \mathrm{ml}\right)$. Animals infected with SIVsmE543-3 and SIVsmE543CT\#154 showed similar chronic phase plasma viral RNA load ranging from $10^{4}$ to $10^{7}$ copies/ $\mathrm{ml}$ (Figure 9A), except for H890, which controlled systemic viral replication by 6 weeks after infection. This result indicates that introduction of 4 amino acid substitutions in the cytoplasmic tail of gp 41 did not attenuate the viral replication in vivo and did not increase the plasma viral RNA load. All animals showed peak viremia in the CSF at 2 weeks after infection, regardless of potential neurovirulence of the virus with which they were infected, consistent with our previous observation (15). Peak CSF viral RNA loads of the group inoculated with SIVsmE543\#154 were variable $\left(9 \times 10^{2}\right.$ to $3 \times 10^{5}$ copies $\left./ \mathrm{ml}\right)$ compared with group inoculated with SIVsmE543-3 $\left(1 \times 10^{4}\right.$ to $5 \times 10^{5}$ copies/ml), but this variation did not influence the CSF viral RNA load in the chronic phase of infection (Figure 9B). The CSF viral RNA loads of animals inoculated with SIVsmE543-3 declined to undetectable levels by 8 weeks after infection. Detectable viral loads were observed in all SIVsmE543-3 animals at various time points beyond 8 weeks after infection but never increased over $10^{2}$ copies/ml thereafter. The group infected with SIVsmE543CT\#154 showed similar kinetics with the SIVsmE543-3-infected group up to 12 weeks after infection. However, their CSF viral RNA load started diverging from the SIVsmE543-3-infected group around 16 weeks after infection. CSF viral load increased gradually and was 

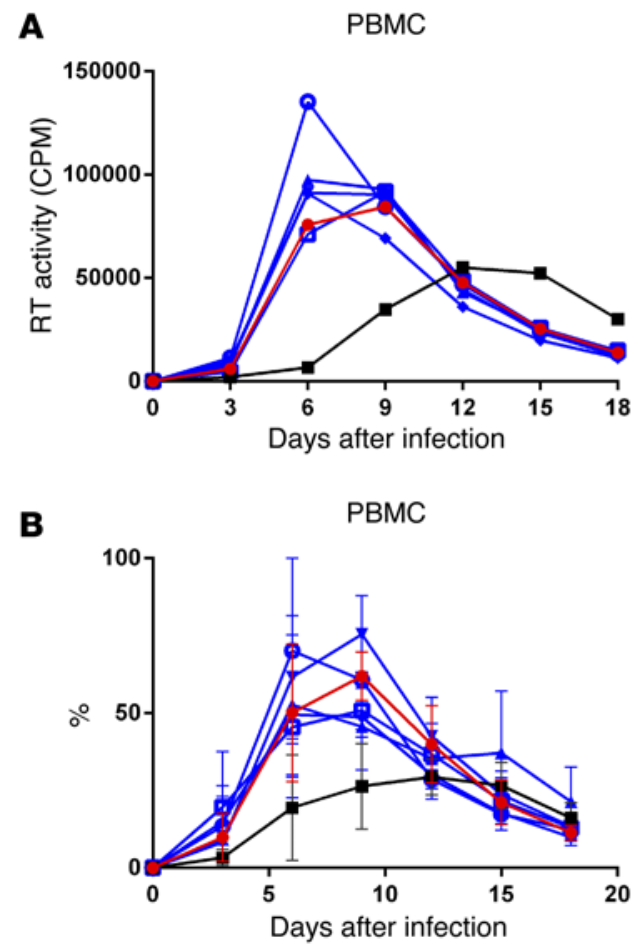

MDM

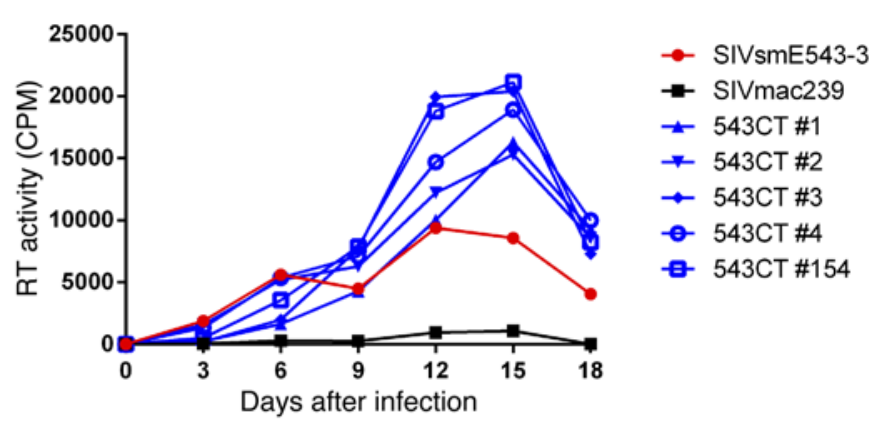

MDM

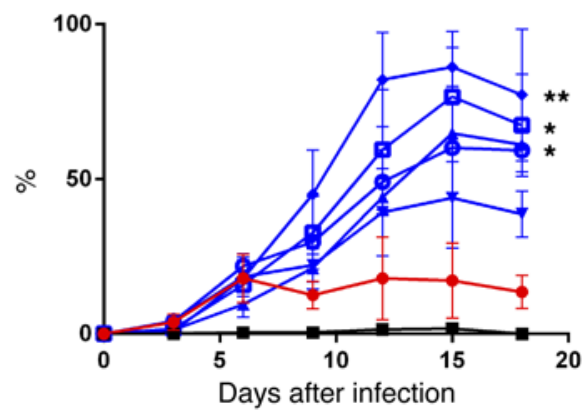

Figure 4. Influence of mutations on in vitro replication capacity. (A) Replication kinetics of SIVsmE543CT\#1, 543CT\#2, 543CT\#3, 543CT\#4, and 543CT\#154 on PBMCs and MDMs. Three independent experiments were conducted with PBMC and MDM obtained from donors RhDCCW and RhDCRG. A representative experiment is shown (donor RhDCCW). (B) Means of replication efficiency normalized as percentage of maximal replication activity reached for different cell preparations infected with indicated set of viruses. ${ }^{*} P \leq 0.05$ and ${ }^{* *} P \leq 0.01$, 2-way ANOVA (replication kinetics of SIVsm mutants compared with SIVsmE543).

maintained at approximately $10^{3}$ copies $/ \mathrm{ml}$ in all animals (H888, H889, H891, H892, and H893) at multiple time points, except for the animal (H890) that had controlled the viral replication for unknown reasons. This tendency became more obvious with time, as 2 animals showed elevations of CSF viral RNA load at 32 weeks after infection (H888, $1 \times 10^{5}$ copies $/ \mathrm{ml} ; \mathrm{H} 889,4 \times 10^{4}$ copies $/ \mathrm{ml}$ ) to levels we have previously reported as associated with development of SIV encephalitis (SIVE) (Figure 9B).

\section{Discussion}

While the effect of BST-2 on virus replication in vitro is reasonably well understood, there is very limited information on the role of BST-2 in influencing virus replication in vivo. To date, there are only 2 reports that suggest that BST-2 antagonism may be critical for viral pathogenesis and in vivo replication of HIV/SIV. One report utilizing humanized mice demonstrated that lack of BST-2 antagonism enhances viral replication in the acute phase of infection (37). Another report demonstrated the importance of BST-2 antagonism for SIV replication in rhesus macaques (29). Serra-Moreno and colleagues demonstrated that SIVmac239 $\Delta n e f$, which is widely used as an attenuated live vaccine, gained pathogenicity in vivo by accumulating compensatory mutations in the cytoplasmic tail of gp 41 to allow the Env protein to antagonize host BST-2. Interestingly, these compensatory mutations were located in a similar region of gp 41 as the mutations identified in our current study. In fact, we found that SIVsmE543-3 exclusively utilizes its Env protein rather than
Nef to antagonize BST-2. In addition, we found that even the SIVmac239 Env protein was able to enhance viral release at the same level as its respective Nef protein, suggesting that viruses have evolved redundant mechanisms to antagonize BST-2. This finding is reminiscent of a previous report on HIV-1 AD8 where both viral protein unique $(\mathrm{Vpu})$ and Env protein were found to antagonize BST-2 restriction in macrophages (38). We previously reported that HIV-2 was derived by cross-species transmission of SIVsm from sooty mangabey (39). In that regard, our observation that SIVsm employs Env to antagonize BST-2 has evolutionary significance, since HIV-2 is well known to use its Env protein to antagonize BST-2, as well (40). Therefore, it has been speculated that SIVsm has adapted to antagonize hBST-2 by its Env protein instead of Nef protein during interspecies transmission. In fact, our results suggest that SIVsm strains, which are the direct ancestors of HIV-2, can utilize the same protein to antagonize the host BST-2, indicating that BST-2 did not restrict zoonotic transmission to humans. Indeed, SIVsmE543-3 Env protein can antagonize hBST-2 with efficiency similar to its antagonism of rBST-2 (data not shown). The dogma for accessory genes antagonizing BST-2 has been that HIV-1 utilizes Vpu (30, 31), HIV-2 utilizes Env (40), and SIV utilizes Nef protein (32) in their respective hosts. However, recent reports on BST-2 antagonism suggest that this is not always the case. For instance, $\mathrm{Vpu}^{+}$SIVs such as SIV from greater spot-nosed monkeys (SIVgsn) can utilize their Nef protein to antagonize BST-2 (41), some HIV-2 Env variants also fail to antagonize BST-2 (35), and HIV-1 group O - which lacks 

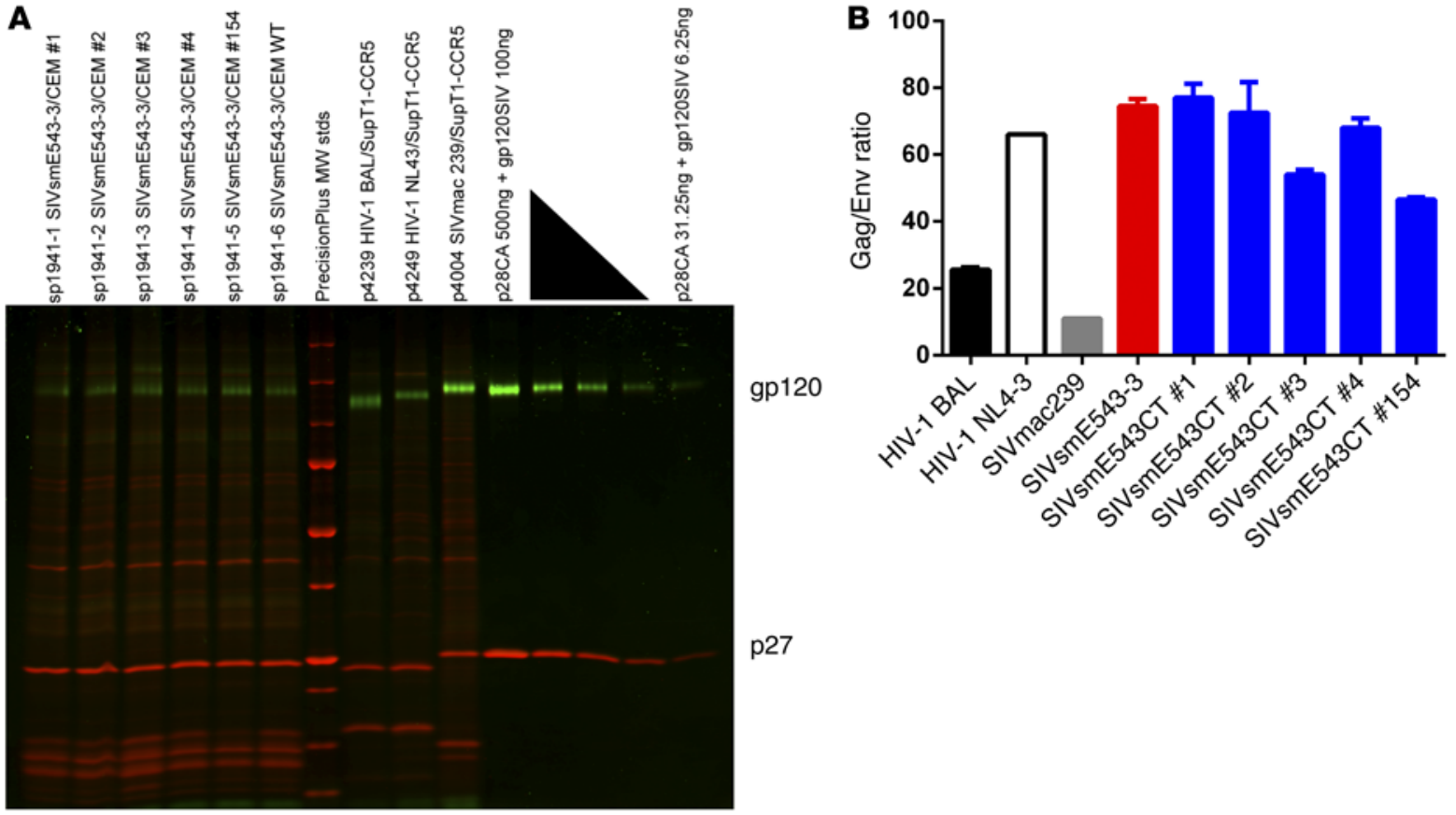

Figure 5. Virion-associated Env content analysis. (A) SDS-PACE gel stained with 2 SYPRO dyes to detect and quantitate glycoprotein (green) and total protein (red). Purified WT SIVsmE543-3 and SIVsmE543CT clones were lysed and run on the same gel with well-characterized reference viruses and serial dilutions of quantified, purified gp120 and p27 for the establishment of standard curves for densitometry analysis. MW stds, molecular mass standards $(\mathrm{kDa})$. A representative of 2 independent experiments is shown. (B) p27/gp120 ratio of WT SIVsmE543-3 and SIVsmE543CT clones, along with well-characterized HIV-1 (BAL and NL-4-3) and SIVmac239. Data represent means \pm SEM from 2 independent analyses.

Vpu expression - antagonizes BST-2 using its Nef protein (42). In addition, we demonstrated in this study that SIVsmE543-3 and SIVmac239 utilize distinct strategies to antagonize BST-2, despite being closely related to each other. Combined, these findings suggest that viral strategies to antagonize BST-2 by HIV-1/SIV are flexible and potentially redundant, indicating the importance of BST-2 antagonism in vivo.

The fact that SIVsmE543-3 can antagonize BST-2 using its Env protein raises the question of the role of BST- 2 in the CNS microenvironment. The neurovirulent virus SIVsm804E was obtained by sequential passage of nonneurovirulent parental virus SIVsmE543-3 through rhesus macaques. One marked difference between these 2 strains of virus is that SIVsm804E shows enhanced replication in macrophages (15). There are numerous reports - including ours that indicate that, in the CNS, HIV-1 and SIV predominantly replicate in myeloid cells, including macrophages $(21,43,44)$. Therefore, it is conceivable that acquisition of the ability to replicate efficiently in macrophages is the result of adaptation to the CNS microenvironment. This is further supported by our previous observations demonstrating that the expression level of BST-2 protein is greater in macrophages than in $\mathrm{T}$ cells (45). Since it is widely accepted that BST-2 antagonism by viral proteins occurs in a dose-dependent manner, either elevated quantity or quality of antagonists may be required to antagonize BST-2 in cells that express high levels of BST-2. In this current study, we have identified 4 mutations (I805T, I828R, T829A, and V878I) in the gp41 cytoplasmic tail of neurovirulent SIVsm804E that conferred enhanced BST-2 antagonism to SIVsmE543-3, as well as enhanced replication in macrophages. Taken together, these observations suggest that higher RT activity in the supernatant of SIVsmE543CT-infected macrophage cultures was the result of enhanced viral release rather than enhanced replication capacity. As described above, SIVmac239 Env protein can also antagonize BST-2 with lower efficiency compared with SIVsmE543 Env protein. Thus, the loss of Nef, for instance, in attenuated SIVmac2394nef conceivably creates the pressure on Env to counteract BST-2 restriction more efficiently. Therefore, the observations with SIVmac239 $\Delta$ nef may actually indicate the enhancement of BST-2 antagonism by Env protein rather than a switch from Nef to Env for BST-2 antagonism, as suggested by Serra-Moreno (29).

Our observation that 4 amino acid substitutions in the cytoplasmic tail of gp41 independently enhance BST-2 antagonism could suggest that these substitutions were the result of the viral adaptations to the CNS microenvironment to counteract BST-2 restriction more efficiently. This speculation is supported by our observation of increasing accumulation of mutations in the viruses (SIVsm445, SIVsm631Br, and SIVsm783Br) isolated during the sequential passages. Mutations were accumulated as passage proceeded, correlating with the increased incidence of neuropathogenesis in infected animals. The fact that multiple independent mutations can increase BST-2 antagonism by Env may indicate that the observed mutations do not directly constitute BST-2 binding sites but more likely induce conformational changes in the Env cytoplasmic tail that increase the ability of Env to interact with BST-2. It is noteworthy that we have previously observed the T829A mutation in an independent brain isolate (46), further supporting the idea that these mutations are strongly associated with viral replication in the CNS. 
A
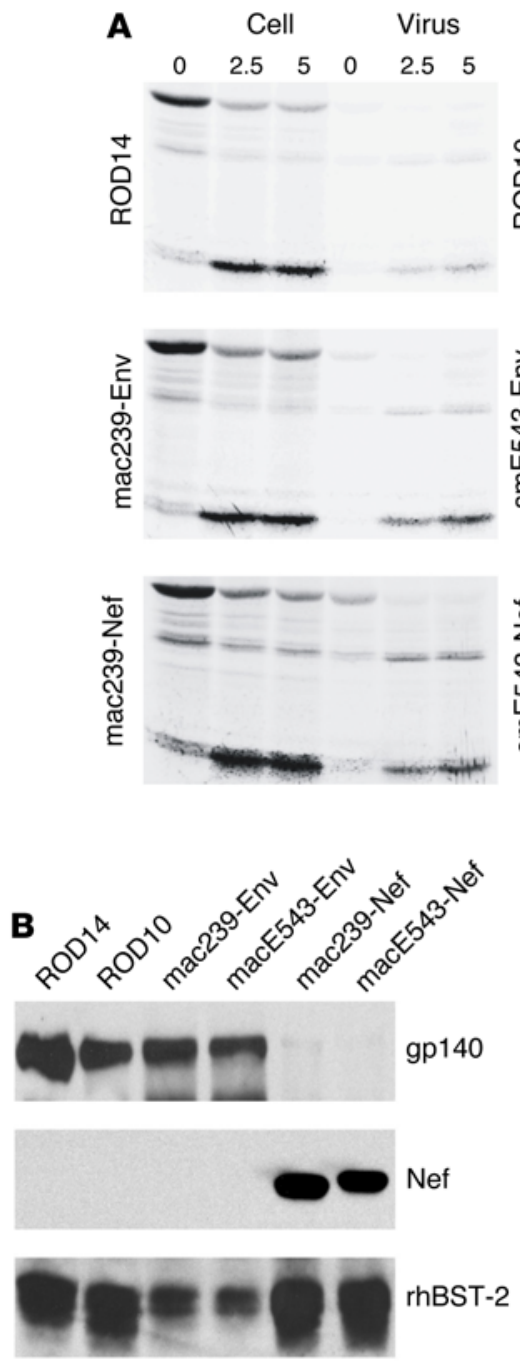

(T) Tubulin
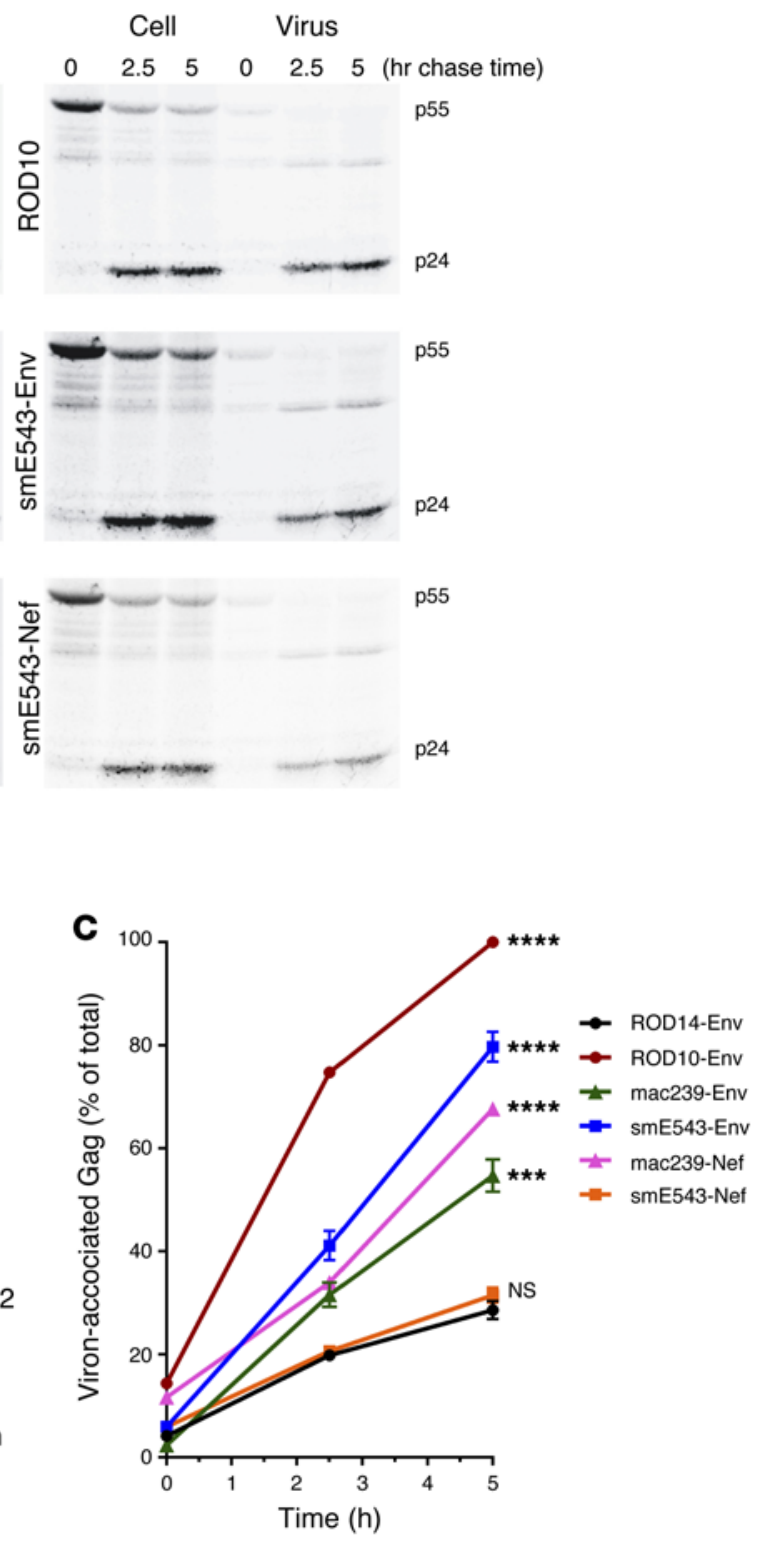

Figure 6. The SIVsm Env glycoprotein enhances HIV-1 particle release. (A) Kinetic analysis of viral particle release by the Vpu-deficient pNL4-3/Udel-1 in the presence of HIV-2 Env, SIV Env, or SIV Nef. 293T cells were transfected with pNL4-3/Udel-1 and rBST-2 DNA together with HIV-2 Env vectors pHA-ROD14Env and pHA-ROD10-Env as controls, as well as vectors for the expression of HA-tagged Envs from SIVmac239 and SIVsmE543 or vectors for the expression of HA-tagged Nef from SIVmac239 and SIVsmE543. Samples were subjected to pulse-chase analysis, and viral proteins recovered by immunoprecipitation were separated by SDS-PAGE. The HIV-1 major Gag proteins p55gag and p24CA are identified on the right. (B) Protein expression for Env, Nef, and rBST-2 was verified by Western blot analysis using cellular $\alpha$-tubulin as a loading control. Representative data from 2 independent experiments are shown. (C) Bands corresponding to the precursor and mature Gag proteins in $\mathbf{A}$ were quantified, and the efficiency of particle release at each time point was calculated and plotted as a function of time. Maximal virus release by ROD10 at the 5-hour time point was defined $100 \%$, and the remaining data points were normalized accordingly. Data represent means \pm SEM from 2 independent analyses. ${ }^{* *} P \leq 0.001$ and ${ }^{* * *} P$ $\leq 0.0001$, 2-way ANOVA (particle release kinetics in the presence of different SIV Env and Nef proteins compared with release by negative control ROD14Env).
Additional support for our hypothesis comes from our in vivo analysis of SIVsm543-3 with 4 amino acid substitutions at the gp41 cytoplasmic tail (SIVsmE543CT\#154). The fact that animals inoculated with SIVsm543CT\#154 showed comparable plasma viral RNA load but higher CSF viral RNA load compared with WT SIVsmE543-3-inoculated animals highlights the importance of BST-2 antagonism in vivo, especially in the CNS microenvironment where myeloid cells are the main target for viral infection and replication. Obviously, long term evaluation of disease outcome and viral loads will be necessary to conclusively identify the Env substitutions as the key determinant of neurovirulence. However, we have observed this pattern of a short period of latency followed by increasing CSF viral load in macaques inoculated with the uncloned neurovirulent strain that subsequently developed neuroAIDS (15).

In summary, the neurovirulent virus SIVsm804E has accumulated mutations in the gp 41 cytoplasmic tail through sequential in vivo passages. Although further analysis is required to assess potential influence of these mutations on other factors, mutations were not associated with increased incorporation of Env into virions but were strongly associated with enhanced BST-2 antagonism that enables efficient viral release from the macrophages. Although the precise mechanisms have yet to be determined, enhanced BST-2 antagonism conferred the ability of virus to replicate robustly in the CNS, as suggested by increased CSF viral load. These observations suggest that BST-2 restriction plays a substnatial role in limiting viral replication in the CNS. Current cART regimens have substantially reduced the incidences of HAD but have not eliminated disease progression in CNS completely. Although SIV infection and disease progression in our nonhuman primate model may not directly reflect the disease progression in HIV-1-infected individuals due to the difference in the BST-2 antagonist utilized, our observations suggest that intervention of BST-2 antagonism by HIV-1 Vpu protein may be used to augment the efficacy of ART to prevent development of HAND. 

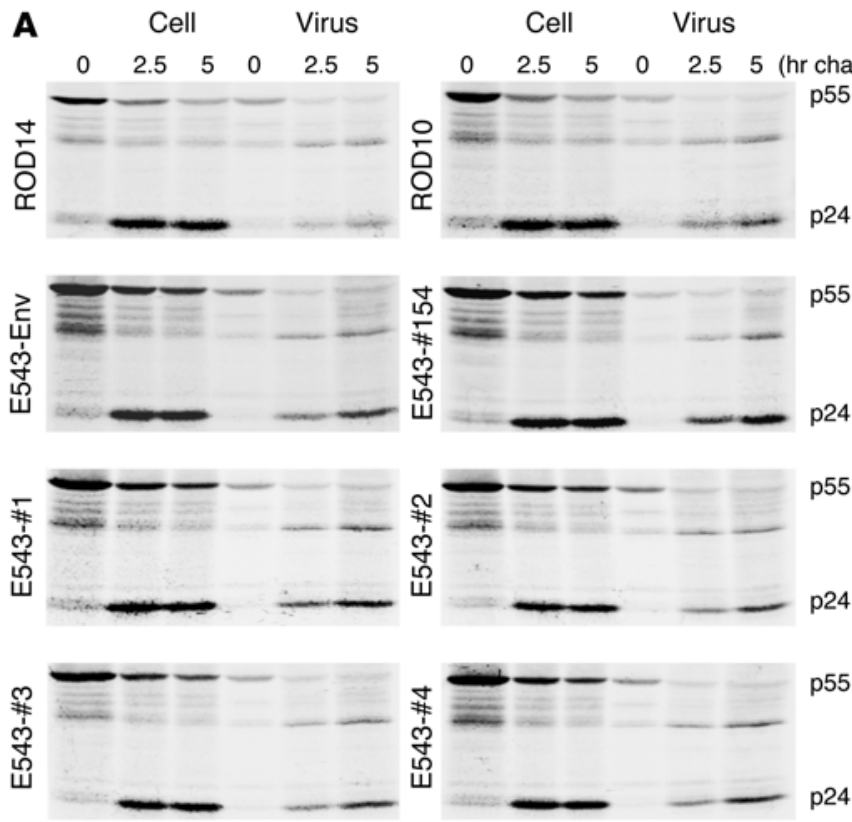

B
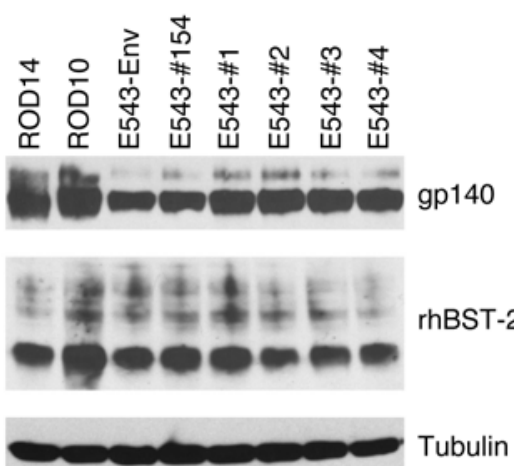
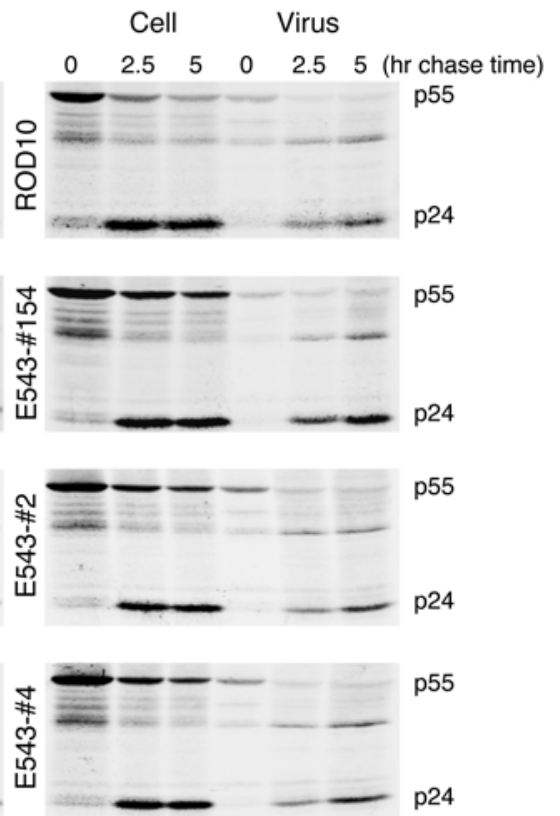

5

55

p24

p55

p24

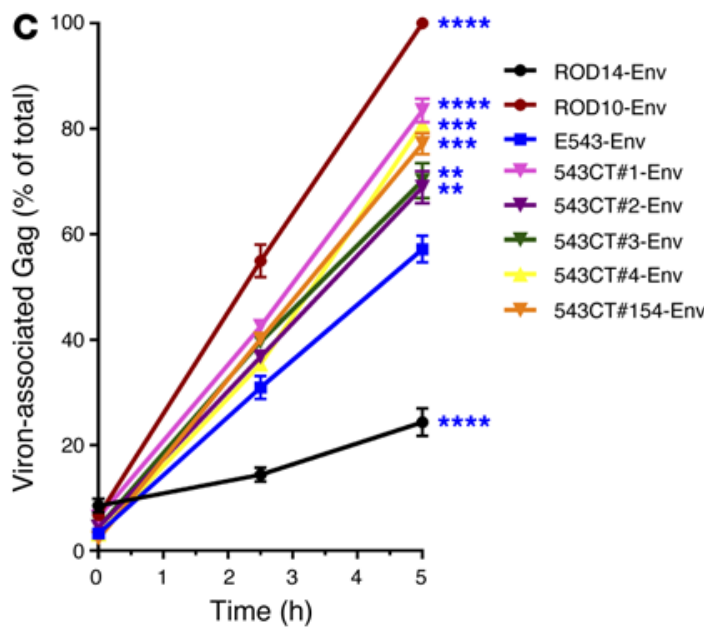

Figure 7. SIVsm Env proteins with mutations in the cytoplasmic tail enhance HIV-1 particle release. (A) Kinetic analysis of viral particle release by the Vpu-deficient pNL4-3/Udel-1 in the presence of HIV-2 Env or SIVsm Env. 293T cells were transfected with pNL43/Udel-1 and rBST-2 DNA together with HIV-2 Env vectors pHA-ROD14-Env and pHA-R0D10-Env as controls, as well as vectors for the expression of HA-tagged Envs from SIVsmE543 WT or mutant SIVsmE543\#1, \#2, \#3, \#4, and \#154.

Samples were subjected to pulse-chase analysis, and viral proteins recovered by immunoprecipitation were separated by SDS-PACE. The HIV-1 major Gag proteins p55gag and p24CA are identified on the right. (B) Protein expression for Env and rBST-2 was verified by Western blot analysis using cellular $\alpha$-tubulin as a loading control. Representative data of 2 independent experiments are shown. (C) Bands corresponding to the precursor and mature Gag proteins in $\mathbf{A}$ were quantified, and the efficiency of particle release at each time point was calculated as in Figure 4 and plotted as a function of time. Bands corresponding to the precursor and mature Gag proteins in $\mathbf{A}$ were quantified, and the efficiency of particle release at each time point was calculated as in Figure 4 and plotted as a function of time. Data represent means \pm SEM calculated from 2 independent experiments. ${ }^{* *} P \leq$ 0.01 ; ${ }^{* *} P \leq 0.001$; and ${ }^{* * * *} P \leq 0.0001$, 2-way ANOVA (particle release kinetics in the presence of different SIVsmE543 mutant Env proteins compared with release by WT SIVsmE543 Env).

\section{Methods}

Construction of SIV clones. For construction of chimeric SIVsmE543-3 containing 3' gp41, nef, and LTR from SIVsm804E (SIVsm804E CL4E4, CL6E6, and CL7E7), viral RNA was isolated from the SIVsm804E viral stock by QIAamp Viral RNA Mini Kit (QIAGEN). The viral RNA was then reverse transcribed by ThermoScript RT-PCR System (Thermo Fisher Scientific) using Gag-R (all primer sequences are listed in Supplemental Table 1) or R-R reverse primers, covering $5^{\prime}$ and 3' LTR sequences. PCR was performed on each resultant cDNA by Platinum Taq Hi Fidelity Kit (Thermo Fisher Scientific) using R-F and Gag-R, or Bgl-F and R-R, respectively. Two PCR products were then mixed at a $1: 1$ ratio and incubated at $95^{\circ} \mathrm{C}$ for 5 minutes before being cooled down to the room temperature to allow annealing of the 2 products at the shared R region. A complete LTR with partial env sequence on the 5 ' side and primer binding site on the 3 ' side was obtained by overlapping PCR of above product by Platinum Taq Hi Fidelity Kit using Bgl-F and Gag-R as primers. This product was then TA cloned into the pCR4-TOPO vector using a TA cloning kit (Thermo Fisher Scientific), resulting in 1LTR TOPO, and sequenced. To create 2LTR puc19 with unique restriction sites that allow the rest of the coding regions to be transferred, 1LTR TOPO clones were digested with NdeI and NarI restriction enzymes and transferred into puc19 vector (New England Biolabs), resulting in 1LTR puc19. 1LTR TOPO vector was digested with EcoRI and NarI, and the fragment with LTR sequence was introduced into the region between EcoRI and SmaI of 1LTR puc19 vector to create a 2LTR puc19 vector. The WT SIVsmE543-3 fulllength plasmid was digested with NarI and BglII restriction enzymes and inserted into the region between the same restriction sites of 2LTR puc19 vector to obtain full-length vectors.

To obtain SIVsm543 804CTN (SIVsmE543 backbone with 3' gp41 and neffrom SIVsm804E CL7E7) and SIVsmE543 804CT (SIVsmE543 backbone with $3^{\prime}$ gp41 from SIVsm804E CL7E7), 2 sets of 2 PCRs each were conducted using Herculase II Fusion DNA polymerase (Aligent Technologies) and SIVsm804E CL7E7 or SIVsmE543-3 fulllength plasmids as a template. For the first reaction, primers Bgl-F and nefend-R were used for amplification of the $3^{\prime}$ end of gp41 and nef, or primers env10651-F and nefstart-R were used for amplification of the $3^{\prime}$ end of gp41 from SIVsm804E CL7E7. The primers Hind-R and 


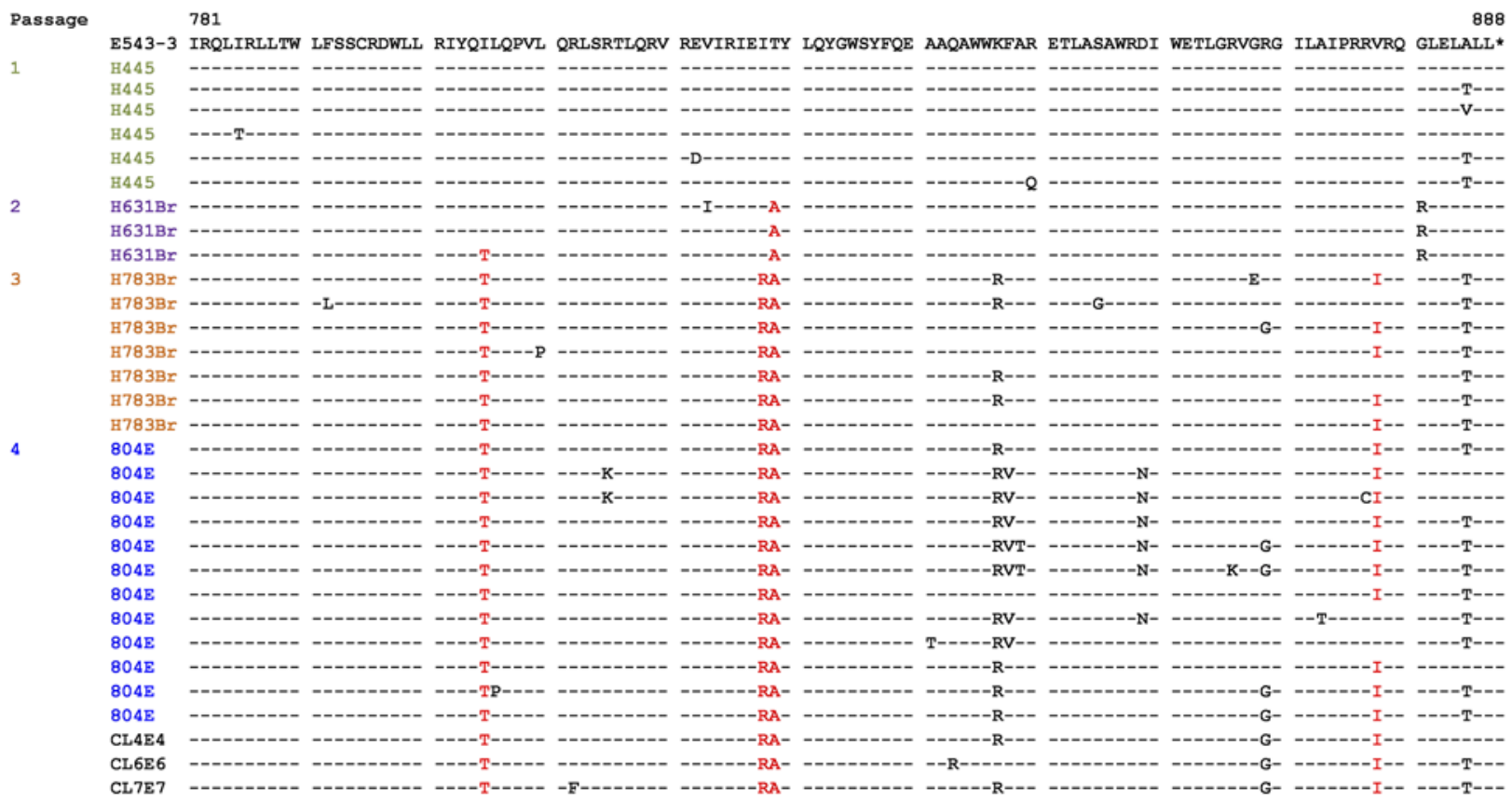

Figure 8. 3' gp41 amino acid sequences of historical viral stocks isolated during sequential in vivo passages. Four amino acid substitutions identified to enhance BST-2 antagonism in this study are shown in red letters. Asterisks indicate stop codons.

nefend-F were used to amplify $3^{\prime}$ LTR; nefstart-F and Hind-R primers were used to amplify nef and 3' LTR from SIVsmE543-3 in the second reaction. The 2 corresponding overlapping PCR products were annealed as described above and amplified by Platinum Taq Hi Fidelity PCR using Bgl-F and Hind-R primers. The resulting PCR product was then digested with HindIII and BglII restriction enzymes, and the fragment with chimeric gp $41 /$ nef/LTR sequence was introduced back into the corresponding region of SIVsm804E CL7E7.

Construction of SIVsmE543 with amino acid substitutions in the gp41 cytoplasmic tail. To obtain SIVsmE543CT\#1, 543CT\#2, 543CT\#3, and $543 \mathrm{CT} \# 4,8$ sets of 2-PCR reactions were conducted to introduce mutations into the cytoplasmic tail of WT SIVsmE543-3. A fragment containing the gp41 cytoplasmic tail was amplified by Herculase II Fusion DNA polymerase using mismatch primers listed in Supplemental Table 2. Reactions were paired with matched Hind-R or Bgl-F primers, as appropriate. Each pair of PCR products was then annealed and used as a template for the second round of PCR using Platinum Taq Hi Fidelity Kit using Bgl-F and Hind-R primers. The resulting fragments with mutated gp41 cytoplasmic tails were TA cloned into the pCR4-TOPO vector using a TA cloning kit. The resulting constructs were digested with HindIII and BglII restriction enzymes and substituted into the corresponding region of WT SIVsmE543-3 plasmid.

To obtain SIVsmE543CT\#154, site-directed mutagenesis was conducted as explained above. The first PCR reaction was conducted using SIVsmE543-3 plasmid as a template. Two subsequent (2nd and 3rd) PCRs were performed using the PCR product from the previous reaction. All final constructs were verified by sequence analysis.

Viral stocks. Viral stocks were obtained by transfection of 293T cells (AIDS reagent program, catalog 103) with full-length molecular clones using Fugene 6 Transfection Reagent (Promega). Supernatants were collected 48 hours after transfection and cryopreserved in $-80^{\circ} \mathrm{C}$ until use. SIVsm804E viral stock was prepared as described previously (15). Briefly, a single cell suspension was prepared from the brain of a rhesus macaque that had developed severe SIVE by the time of necropsy. This single cell suspension was then cocultured with peripheral blood mononuclear cells (PBMCs) isolated from SIV-naive rhesus macaques. Culture supernatants were collected every 3 days, up to 18 days, and the stock with the highest RT activity was cryopreserved at $-80^{\circ} \mathrm{C}$ as a virus stock. The $50 \%$ tissue culture infectious dose $\left(\mathrm{TCID}_{50}\right)$ of all virus stocks was measured by TZM-bl assay as described elsewhere (47).

Sequence analysis. Env sequences of SIVsmH445, SIVsmH631Br, and SIVsmH783Br viral stocks were characterized in the previous study by the single-genome amplification method $(15,48)$. The Env sequence of the SIVsm804E viral stock was characterized as previously described (14). Briefly, total RNA was extracted from the viral stock, and reverse transcription was performed by ThermoScript RT-PCR system (Thermo Fisher Scientific) using the primer 9341-R (See Supplemental Table 1 for primer sequences). PCR was performed on the cDNA product by Platinum Taq Polymerase High Fidelity Kit using the following primers: 6463-F and 9341-R. PCR product was then TA cloned into the TOPO vector and sequenced.

Infection assays. Virus replication of the isolates was evaluated in rhesus macaque PBMCs and MDMs as described previously (14, 15). PBMCs from SIV-naive, healthy rhesus macaques were separated from whole blood, stimulated with $5 \mu \mathrm{g}$ of phytohemagglutinin (PHA) per ml and 10\% IL-2 (Advanced Biotechnologies) for 3 days, and maintained in RPMI 1640 medium containing 10\% FCS and 10\% IL-2. Rhesus macaque MDMs were obtained from rhesus macaque PBMCs as previously described (15). Briefly, fresh PBMCs were incubated with anti-nonhuman-primate CD14 magnetic beads (Miltenyi Biotec) and positively selected with MACS separation columns (Miltenyi Biotec). A total of $3 \times 10^{5}$ cells per well of $\mathrm{CD} 14^{+}$cells 
A

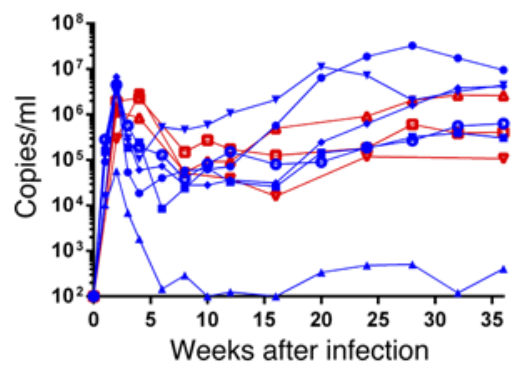

B

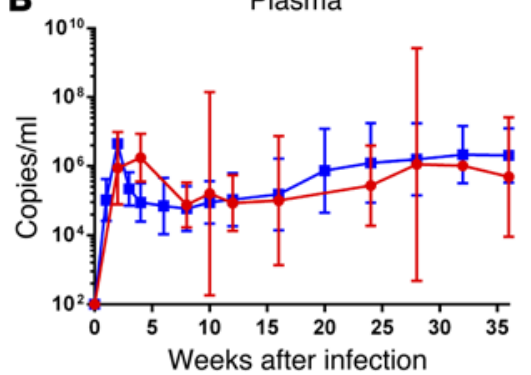

CSF

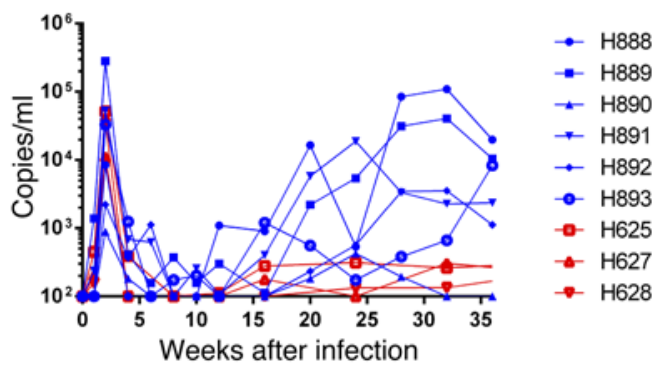

CSF

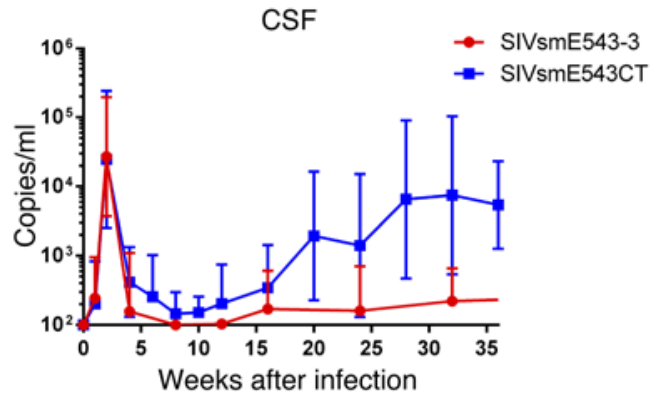

Figure 9. In vivo replication of

SIVsmE543CT\#154. (A) Plasma and CSF vira RNA loads. Red lines indicate SIVsmE543-3infected animals, and blue line indicate SIVsmE543CT\#154-infected animals. (B) Ceometric mean with $95 \% \mathrm{Cl}$ of plasma and CSF viral loads. $\mathrm{H} 890$ is excluded from the group, since this animal controlled viral replication systemically for unknown reasons. were cultured in a 48-well plate for 4 days in RPMI 1640 containing $10 \%$ FCS, $10 \%$ human serum type AB (Sigma-Aldrich), and $20 \mathrm{ng} / \mathrm{ml}$ of macrophage CSF (R\&D Systems). Cells were washed 2 times with HBSS and cultured in fresh medium for 3 additional days. PBMCs (5 $\times 10^{5} /$ well) were dispensed into a 48 -well plastic plate and then inoculated with each virus at a MOI of 0.01. MDMs were incubated with virus at an MOI of 0.01 for 1 hour and then washed twice with HBSS and cultured in fresh medium. Virion-associated RT activity of the culture supernatant was monitored periodically, as we have described previously (49). To evaluate differences between replication kinetics of SIVsmE543 and constructed chimeric clones and mutants, 3 independent sets of experiments were conducted with PBMC and MDM obtained from donors RhDCCW and RhMO3. The obtained data were normalized as percentage of maximal value reached inside every independent experiment.

Protein analysis. Quantitative measurements of viral p27 capsid (CA) and gp120 protein in virions for determinations of Gag/Env ratio were determined using dual-color fluorescent protein gel analysis as previously described $(27,28)$. Briefly, SIVsmE543-3 and SIVsmE543CT clones were expanded in CEM cells, concentrated and purified, and examined by SDS-PAGE. Well-characterized reference preparations of infection-derived HIV-1 BAL, HIV-1 NL4-3, and SIVmac239 cultured in Human SupT1-CCR5 T lymphoblastoid cells (provided by Jim Hoxie, University of Pennsylvania, Philadelphia, Pennsylvania, USA) were included in the analysis as controls. Total protein was stained using SYPRO Ruby (Thermo Fisher Scientific), allowing for determination of p27 content, and glycoprotein was stained with SYPRO Pro-Q Emerald (Thermo Fisher Scientific), allowing for determination of gp120 content. Virion p27/gp120 contents were quantitated using linear regression analysis by generating densitometry standard curves with dilution series of purified p27 and pg120 of known concentration. Gag/Env ratio for each samples were then calculated.

Assessment of viral particle release. pHA-ROD10 and pHA-ROD14-Env expression vectors were constructed by adding a HA-tag to the C-terminus of the pCM10 and pCM14 plasmid as described previously (35). pHA-mac239-Env (SIVmac239), pHAsmE543-Env (SIVsmE543-3), pHA-E543\#1 (SIVsmE543CT\#1), pHA-E543\#2 (SIVsmE543CT\#2), pHA-E543\#3 (SIVsmE543CT\#3), pHA-E543\#4 (SIVsmE543CT\#4), and pHA-E543\#154 (SIVsmE543CT\#154) Env expression constructs were generated by cloning the PCR-amplified full-length Env sequences of respective viral strains via the flanking XbaI and XhoI restriction sites into the corresponding sites in pCM10. The pHA-mac239-Nef (SIVmac239) and pHA-smE543-Nef (SIVsmE543-3) nef expression constructs were generated by cloning the PCR-amplified full-length nef sequences of respective viral strains flanked by EcoRI and XhoI restriction sites into the corresponding sites of pCAG-HA vector (Addgene).

Pulse chase and immunoprecipitation assays were performed, as described previously, with some modifications (40). Briefly, $293 \mathrm{~T}$ cell were cotransfected with $\mathrm{rBST}-2$ expression vector, Vpu deleted pNL4-3 plasmid (50), and one of the respective HA-tagged Env expression vectors (ROD10 env, ROD14 env, SIVmac239 env, SIVmac239 nef, SIVsmE543-3 env, SIVsmE543-3 nef, SIVsmE543CT\#1 env, SIVsmE543CT\#2 env, SIVsmE543CT\#3 env, SIVsmE543CT\#4 env, or SIVsmE543CT\#154 env) using Lipofectamine with Plus Reagent (Thermo Fisher Scientific). Transfected $293 \mathrm{~T}$ cells were pulse labeled with $\left[{ }^{35} \mathrm{~S}\right]$-translabel (Perkin Elmer) for 30 minutes and chased at $37^{\circ} \mathrm{C}$ in $1 \mathrm{ml}$ of prewarmed DMEM-FBS for $0,2.5$, and 5 hours. At each time point, cells were collected and lysed in $400 \mu$ l of Nonidet P-40-deoxycholate (NP-40-DOC) buffer (20 mM Tris-HCL [pH 8], 120 mM NaCl, 2 mM EDTA, 0.5\% DOC, $1 \% \mathrm{NP}-40$ ). The culture supernatants were filtered through $0.45 \mathrm{um}$ pore-size cellulose acetate Spin-X centrifuge tube filters (SigmaAldrich) to remove remaining cells and cell debris. Virus particles were then pelleted from cell-free supernatants in a microcenrifuge $\left(4^{\circ} \mathrm{C} ; 90 \mathrm{~min} ; 16,000 \mathrm{~g}\right)$. Pelleted virions were lysed in $400 \mu \mathrm{l}$ of NP-40-DOC buffer. Cell lysates were precleared by incubation at $4^{\circ} \mathrm{C}$ for 1 hour with protein A-Sepharose beads (Sigma-Aldrich) and immunoprecipitated with HIV IgG (NIH Research and Reference reagent program [catalog 3957]). Immunoprecipitates were solubi- 
lized by heating in sample buffer containing $2 \%$ SDS, $1 \%$ mercaptoethanol, $1 \%$ glycerol, and $65 \mathrm{mM}$ Tris-HCL (pH 6.8) and separated by SDS-PAGE using $12 \%$ polyacrylamide gels. Gels were fixed, incubated for 20 minutes in $1 \mathrm{M}$ salicylic acid (Sigma-Aldrich), and dried. Radioactive bands were visualized by fluorography using Bio-Max MR films (Kodak). Quantitation of the relevant bands was performed with a Fujix BAS 2000 Bio-Image Analyzer. The efficiency of particle release at each time point was calculated by dividing the amount of Gag proteins present in the pelleted virus fraction by the total of celland virus-associated Gag proteins. The ratio of virion-associated versus total Gag protein was then plotted as a function of time. For this purpose, the data from 2 independent experiments were normalized by taking a maximal release at a 5 -hour point in the presence of ROD10 as 100\%.

In vivo replication of SIVsmE543CT\#154. Naive Indian origin rhesus macaques were prescreened for TRIM $5 \alpha$ genotype, and 9 animals with moderately susceptible TFP/Q were selected for this study. Six animals were i.v. inoculated with 500 TCID $_{50}$ of SIVsmE543CT\#154, and the remaining 3 animals were inoculated with $1,000 \mathrm{TCID}_{50}$ of SIVsmE543-3. Plasma viral RNA loads were monitored periodically. Geometric means with 95\% CI of viral loads were calculated with GraphPad Prism software. H890 was excluded from this calculation, since systemic viral replication was controlled beyond 6 weeks after infection for unknown reasons.

Statistics. To evaluate statistical significance of observed differences in replication kinetics of constructed viruses compared with replication kinetics of SIVsmE543, or differences in viral particle release kinetics in the presence of different SIV Nef or Env proteins, the repeated measures 2-way ANOVA with a post-hoc multiple comparisons by the unprotected Fisher's test were performed by GraphPad Prism 6 for Windows, Version 6.04. Significance is denoted as $P<0.05$.

Study approval. All animals were housed in accordance with the American Association for Accreditation of Laboratory Animal Care standards. The investigators adhered to the Guide for the Care and Use of Laboratory Animals (National Academies Press. 2011.) and to NIAID Animal Care and Use Committee-approved protocols.

\section{Author contributions}

$\mathrm{KM}$ designed the study, performed experiments, analyzed the data, and wrote the paper. VMH and KS designed the study and wrote the paper. CYC performed experiments and analyzed the data. EC, DJR, FW, RJP, and SW performed experiments. JDL and ABW supervised experiments. IO conducted statistical analysis.

\section{Acknowledgments}

We thank Heather Cronise, Joanne Swerzyck, and Richard Herbert at the NIH Animal Center for excellent care of study animals. This work was supported with federal funds from the intramural program of the NIAID, NIH, and with federal funds from the National Cancer Institute, NIH (contract HHSN261200800001E).

Address correspondence to: Vanessa M. Hirsch, LMM, NIAID, NIH, Building 4, Room B1-41, 4 Center Drive, Bethesda, Maryland 20892, USA. Phone: 301.496.0559; E-mail: vhirsch@niaid.nih.gov.
1. Price RW, Brew B, Sidtis J, Rosenblum M, Scheck AC, Cleary P. The brain in AIDS: central nervous system HIV-1 infection and AIDS dementia complex. Science. 1988;239(4840):586-592.

2. Baldeweg T, Catalan J, Lovett E, Gruzelier J, Riccio M, Hawkins D. Long-term zidovudine reduces neurocognitive deficits in HIV-1 infection. AIDS. 1995;9(6):589-596.

3. Reinvang I, Froland SS, Karlsen NR, Lundervold AJ. Only temporary improvement in impaired neuropsychological function in AIDS patients treated with zidovudine. AIDS. 1991;5(2):228-229.

4. Heaton RK, et al. HIV-associated neurocognitive disorders persist in the era of potent antiretroviral therapy: CHARTER Study. Neurology. 2010;75(23):2087-2096.

5. Sklar PA, et al. Prevalence and clinical correlates of HIV viremia ('blips') in patients with previous suppression below the limits of quantification. AIDS. 2002;16(15):2035-2041.

6. Greub G, et al. Intermittent and sustained low-level HIV viral rebound in patients receiving potent antiretroviral therapy. AIDS. 2002;16(14):1967-1969.

7. Letendre S, et al. Validation of the CNS penetration-effectiveness rank for quantifying antiretroviral penetration into the central nervous system. Arch Neurol. 2008;65(1):65-70.

8. Canestri A, et al. Discordance between cerebral spinal fluid and plasma HIV replication in patients with neurological symptoms who are receiving suppressive antiretroviral therapy. Clin Infect Dis. 2010;50(5):773-778.

9. Eden A, et al. HIV-1 viral escape in cerebrospinal fluid of subjects on suppressive antiretroviral treatment. JInfect Dis. 2010;202(12):1819-1825.

10. Eden A, Price RW, Spudich S, Fuchs D, Hagberg L, Gisslen M. Immune activation of the central nervous system is still present after $>4$ years of effective highly active antiretroviral therapy. Jinfect Dis. 2007;196(12):1779-1783.

11. Yilmaz A, Price RW, Spudich S, Fuchs D, Hagberg L, Gisslen M. Persistent intrathecal immune activation in HIV-1-infected individuals on antiretroviral therapy. J Acquir Immune Defic Syndr. 2008;47(2):168-173.

12. Akay $\mathrm{C}$, et al. Antiretroviral drugs induce oxidative stress and neuronal damage in the central nervous system. J Neurovirol. 2014;20(1):39-53.

13. Robertson K, Liner J, Meeker RB. Antiretroviral neurotoxicity. J Neurovirol. 2012;18(5):388-399.

14. Matsuda K, et al. Laser capture microdissection assessment of virus compartmentalization in the central nervous systems of macaques infected with neurovirulent simian immunodeficiency virus. JVirol. 2013;87(16):8896-8908.

15. Matsuda K, et al. Characterization of simian immunodeficiency virus (SIV) that induces SIV encephalitis in rhesus macaques with high frequency: role of TRIM5 and major histocompatibility complex genotypes and early entry to the brain. JVirol. 2014;88(22):13201-13211.

16. Thompson KA, et al. Decreased neurotropism of nef long terminal repeat (nef/LTR)-deleted simian immunodeficiency virus. J Neurovirol. 2003;9(4):442-451.

17. Mankowski JL, et al. Pathogenesis of simian immunodeficiency virus encephalitis: viral determinants of neurovirulence. J Virol. 1997;71(8):6055-6060.

18. Flaherty MT, Hauer DA, Mankowski JL, Zink MC, Clements JE. Molecular and biological characterization of a neurovirulent molecular clone of simian immunodeficiency virus. J Virol. 1997;71(8):5790-5798.

19. van Marle G, et al. Human immunodeficiency virus type 1 Nef protein mediates neural cell death: a neurotoxic role for IP-10. Virology. 2004;329(2):302-318.

20. Chompre G, Cruz E, Maldonado L, Rivera-Amill V, Porter JT, Noel RJ Jr. Astrocytic expression of HIV-1 Nef impairs spatial and recognition memory. Neurobiol Dis. 2013;49:128-136.

21. Koenig S, et al. Detection of AIDS virus in macrophages in brain tissue from AIDS patients with encephalopathy. Science. 1986;233(4768):1089-1093.

22. Schnell G, Joseph S, Spudich S, Price RW, Swanstrom R. HIV-1 replication in the central nervous system occurs in two distinct cell types. PLoS Pathog. 2011;7(10):e1002286.

23. Puffer BA, et al. CD4 independence of simian immunodeficiency virus Envs is associated with macrophage tropism, neutralization sensitivity, and attenuated pathogenicity. J Virol. 2002;76(6):2595-2605.

24. Bhatia AK, Kaushik R, Campbell NA, Pontow SE, Ratner L. Mutation of critical serine residues in HIV-1 matrix result in an envelope incorporation defect which can be rescued by truncation of the gp 41 cytoplasmic tail. Virology. 2009;384(1):233-241. 
25. Wyss S, Dimitrov AS, Baribaud F, Edwards TG, Blumenthal R, Hoxie JA. Regulation of human immunodeficiency virus type 1 envelope glycoprotein fusion by a membrane-interactive domain in the gp41 cytoplasmic tail. J Virol. 2005;79(19):12231-12241.

26. Yuste E, Johnson W, Pavlakis GN, Desrosiers RC. Virion envelope content, infectivity, and neutralization sensitivity of simian immunodeficiency virus. J Virol. 2005;79(19):12455-12463.

27. Del Prete GQ, et al. Comparative characterization of transfection- and infection-derived simian immunodeficiency virus challenge stocks for in vivo nonhuman primate studies.J Virol. 2013;87(8):4584-4595.

28. Louder MK, et al. HIV-1 envelope pseudotyped viral vectors and infectious molecular clones expressing the same envelope glycoprotein have a similar neutralization phenotype, but culture in peripheral blood mononuclear cells is associated with decreased neutralization sensitivity. Virology. 2005;339(2):226-238.

29. Serra-Moreno R, Jia B, Breed M, Alvarez X, Evans DT. Compensatory changes in the cytoplasmic tail of gp 41 confer resistance to tetherin/BST- 2 in a pathogenic nef-deleted SIV. Cell Host Microbe. 2011;9(1):46-57.

30. Neil SJ, Zang T, Bieniasz PD. Tetherin inhibits retrovirus release and is antagonized by HIV-1 Vpu. Nature. 2008;451(7177):425-430.

31. Van Damme N, et al. The interferon-induced protein BST-2 restricts HIV-1 release and is downregulated from the cell surface by the viral $\mathrm{Vpu}$ protein. Cell Host Microbe. 2008;3(4):245-252.

32. Zhang F, et al. Nef proteins from simian immunodeficiency viruses are tetherin antagonists. Cell Host Microbe. 2009;6(1):54-67.

33. Gupta RK, et al. Simian immunodeficiency virus envelope glycoprotein counteracts tetherin/BST-2/CD317 by intracellular sequestration. Proc Natl Acad Sci U S A. 2009;106(49):20889-20894.

34. Bour SP, Aberham C, Perrin C, Strebel K. Lack of effect of cytoplasmic tail truncations on human immunodeficiency virus type 2 ROD env particle release activity. J Virol. 1999;73(1):778-782.

35. Bour S, Akari H, Miyagi E, Strebel K. Naturally occurring amino acid substitutions in the HIV-2 ROD envelope glycoprotein regulate its ability to augment viral particle release. Virology. 2003;309(1):85-98.

36. Jia B, et al. Species-specific activity of SIV Nef and HIV-1 Vpu in overcoming restriction by tetherin/BST2. PLoS Pathog. 2009;5(5):e1000429.

37. Sato K, et al. Vpu augments the initial burst phase of HIV-1 propagation and downregulates BST2 and CD4 in humanized mice. J Virol. 2012;86(9):5000-5013.

38. Schubert U, Bour S, Willey RL, Strebel K. Regulation of virus release by the macrophage-tropic human immunodeficiency virus type 1 AD8 isolate is redundant and can be controlled by either Vpu or Env. J Virol. 1999;73(2):887-896.

39. Hirsch VM, Olmsted RA, Murphey-Corb M, Purcell RH, Johnson PR. An African primate lentivirus (SIVsm) closely related to HIV-2. Nature. 1989;339(6223):389-392.

40. Bour S, Strebel K. The human immunodeficiency virus (HIV) type 2 envelope protein is a functional complement to HIV type 1 Vpu that enhances particle release of heterologous retroviruses. J Virol. 1996;70(12):8285-8300.

41. Takeuchi JS, et al. Coevolutionary dynamics between tribe Cercopithecini tetherins and their lentiviruses. Sci Rep. 2015;5:16021.

42. Kluge SF, et al. Nef proteins of epidemic HIV-1 group O strains antagonize human tetherin. Cell Host Microbe. 2014;16(5):639-650.

43. Dang Q, et al. Development of neurological disease is associated with increased immune activation in simian immunodeficiency virus-infected macaques. J Virol. 2012;86(24):13795-13799.

44. Williams KC, et al. Perivascular macrophages are the primary cell type productively infected by simian immunodeficiency virus in the brains of macaques: implications for the neuropathogenesis of AIDS. J Exp Med. 2001;193(8):905-915.

45. Miyagi E, Andrew AJ, Kao S, Strebel K. Vpu enhances HIV-1 virus release in the absence of Bst-2 cell surface down-modulation and intracellular depletion. Proc Natl Acad Sci US A. 2009;106(8):2868-2873.

46. Hirsch V, et al. A molecularly cloned, pathogenic, neutralization-resistant simian immunodeficiency virus, SIVsmE543-3. J Virol. 1997;71(2):1608-1620.

47. Montefiori DC. Measuring HIV neutralization in a luciferase reporter gene assay. Methods Mol Biol. 2009;485:395-405.

48. Keele BF, et al. Low-dose rectal inoculation of rhesus macaques by SIVsmE660 or SIVmac 251 recapitulates human mucosal infection by HIV-1. JExp Med. 2009;206(5):1117-1134.

49. Kuwata T, et al. Infectious molecular clones from a simian immunodeficiency virus-infected rapid-progressor (RP) macaque: evidence of differential selection of RP-specific envelope mutations in vitro and in vivo. $J$ Virol. 2006;80(3):1463-1475.

50. Klimkait T, Strebel K, Hoggan MD, Martin MA, Orenstein JM. The human immunodeficiency virus type 1-specific protein vpu is required for efficient virus maturation and release. J Virol. 1990;64(2):621-629. 